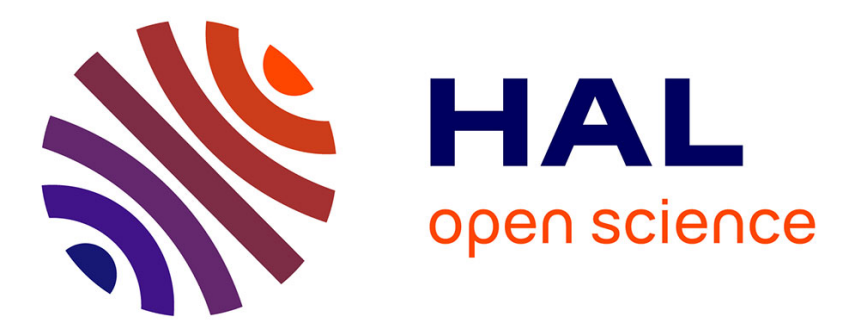

\title{
Light scattering by metallic surfaces with subwavelength patterns
}

Haitao Liu, Philippe Lalanne

\section{To cite this version:}

Haitao Liu, Philippe Lalanne. Light scattering by metallic surfaces with subwavelength patterns. Physical Review B: Condensed Matter and Materials Physics (1998-2015), 2010, 82, pp.115418. 10.1103/PhysRevB.82.115418 . hal-00570642

\section{HAL Id: hal-00570642 \\ https://hal-iogs.archives-ouvertes.fr/hal-00570642}

Submitted on 7 Dec 2015

HAL is a multi-disciplinary open access archive for the deposit and dissemination of scientific research documents, whether they are published or not. The documents may come from teaching and research institutions in France or abroad, or from public or private research centers.
L'archive ouverte pluridisciplinaire HAL, est destinée au dépôt et à la diffusion de documents scientifiques de niveau recherche, publiés ou non, émanant des établissements d'enseignement et de recherche français ou étrangers, des laboratoires publics ou privés. 


\title{
Light scattering by metallic surfaces with subwavelength patterns
}

\author{
Haitao Liu $^{1, *}$ and Philippe Lalanne ${ }^{2}$ \\ ${ }^{1}$ Key Laboratory of Opto-electronic Information Science and Technology, Ministry of Education, \\ Institute of Modern Optics, Nankai University, Tianjin 300071, China \\ ${ }^{2}$ Laboratoire Charles Fabry de l'Institut d'Optique, CNRS, Université Paris-Sud, Campus Polytechnique, \\ 91127 Palaiseau Cedex, France
}

(Received 12 June 2010; published 10 September 2010)

\begin{abstract}
We present a simple and intuitive model for analyzing the optical response of metallic surfaces patterned by arbitrary one-dimensional arrays of subwavelength ( $\operatorname{sub}-\lambda$ ) structures. In the present microscopic approach, the near-field response close to the surface is composed of a linear combination of elementary hybrid waves, which are shown to solely depend on the frequency and to be merely independent of the incident illumination and of the scatterer geometry. The model is tested against fully vectorial computational results obtained for simple systems that can be handled numerically with fully vectorial methods and, in contrast to classical models that solely rely on surface plasmon polaritons, the present model is shown to be highly accurate over a broad spectral range below the plasma frequency of the metal.
\end{abstract}

DOI: 10.1103/PhysRevB.82.115418

PACS number(s): 42.25.Fx, 42.79.Ag, 73.20.Mf, 78.66.-w

\section{INTRODUCTION}

Metallic surfaces patterned with sub- $\lambda$ indentations possess a variety of interesting optical properties, with applications ranging from sensing, photonic, and metamaterial devices, to integrated circuits mixing photonics and electronics. ${ }^{1-3}$ With the progress of nanofabrication, there is an increasing demand for modeling these surfaces. Their properties are very accurately predicted by solving Maxwell's equations with fully vectorial methods, and this can be performed over the entire electromagnetic spectrum, for dimensions from the millimeter down to the nanometer ranges. However, there are some limitations. For instance, except for fully periodic structures, the analysis of surfaces composed of more than a few tens of indentations is extremely demanding in terms of computational resources or impossible. In addition, because of their broad applicability, fully vectorial methods often rely on mathematical and numerical concepts that are distant from our initial intuitive understanding. For instance at optical frequencies, whereas one usually refers to a Huygens-type "microscopic" representation at the level of every individual scatterer for designing sub- $\lambda$ metallic surfaces, ${ }^{1-16}$ assuming that some surface plasmon polaritons (SPPs) initially launched by an illuminated indentation propagate on the metal surface before being further scattered by nearby indentations, modern electromagnetic theories, and computational methods in plasmonics ${ }^{17-19}$ do not explicitly consider the local SPPs and refer to global quantities attached to the whole indentation ensembles. Indeed, having computational tools, theoretical treatments, and intuitive representations that all share the same concepts would largely facilitate further analysis and design of plasmonic and metamaterial devices.

In line with the initial interpretation of Wood's anomalies by Rayleigh and Fano ${ }^{20,21}$ who provided a microscopic theory of metallic gratings and with recent related works $^{13,14,16,22-24}$ on the elaboration of microscopic models aiming at building collective properties of complex systems from the association of individual sub- $\lambda$ entities, we propose an approximate (albeit accurate) microscopy theory of sub- $\lambda$ metallic surfaces. The present approach not only takes into account the SPPs but also another residual wave, called the quasicylindrical wave (quasi-CW) ${ }^{25}$ which is dominant for $\lambda>1000 \mathrm{~nm}$ with noble metals. This allows us to bring an insight into the physics of wave scattering by individual sub- $\lambda$ indentations on metallic surfaces, and to propose a generalized multiple-scattering theory, which sticks to our microscopic representation and provides highly accurate predictions over the entire electromagnetic spectrum below the metal plasma frequency.

The present model relies on two original findings. The first one concerns the field scattered by individual sub- $\lambda$ twodimensional (2D) indentations (invariant in a single $y$ direction) on metallic surfaces. We argue that, for a given frequency, this field forms a fixed entity, called a hybrid wave (HW) hereafter ${ }^{26}$ whose overall shape is independent of the incident illumination and of the exact structure of the sub- $\lambda$ scatterer (Sec. II). The second one concerns the definition of scattering coefficients for the HW (Sec. III), which allows us to propose an intuitive multiple-scattering formalism (Sec. IV). Fundamentally, we show that the near-field of any sub- $\lambda$ metallic surface can be described as a superposition of HWs, generated at every indentation. As shown by comparison with numerical data obtained for simple geometries (such as indentation doublets and periodic arrays of holes) that can be analyzed with fully vectorial methods, the model provides highly accurate predictions and is probably able to faithfully predict the optical properties of a large variety of sub- $\lambda$ metallic surfaces. The results are summarized in Sec. V.

\section{HYBRID WAVES}

Let us start by having a close look at the electromagnetic field scattered by individual sub- $\lambda$ indentations, which lie on an interface between air (relative permittivity $\varepsilon_{d}=1$ ) and gold (the frequency-dependent relative permittivity $\varepsilon_{m}$ is taken from Ref. 27) and which are illuminated by either freespace or bound waves under transverse-magnetic (TM) po- 

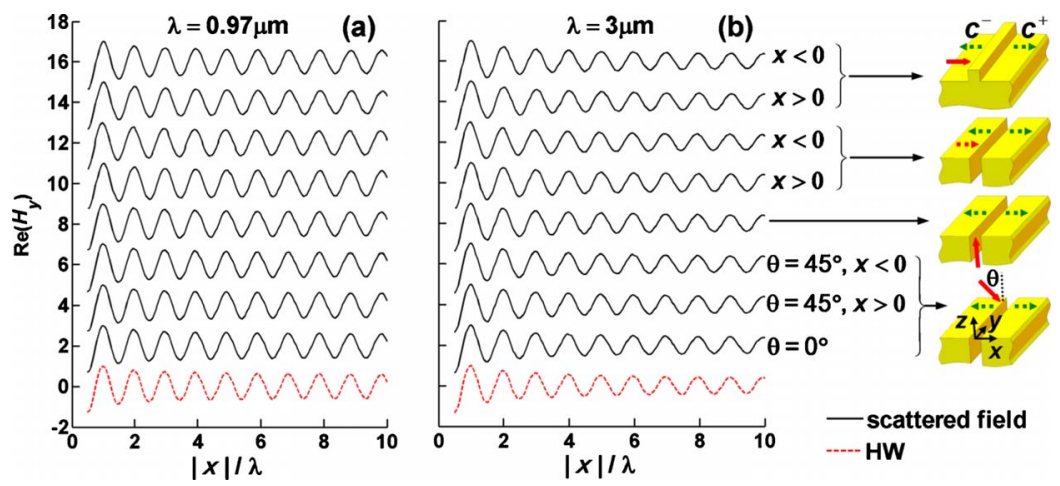

FIG. 1. (Color online) Field scattered by individual 2D sub- $\lambda$ indentations. [(a) and (b)] Magnetic fields $H_{y}$ scattered on the surface (at $z=0)$ for $\lambda=0.97$ and $3 \mu \mathrm{m}$. They are vertically shifted by 2 and normalized such that $H_{y}(|x|=\lambda)=1$. The black solid curves are data calculated with the a-FMM and the two red dashed curves show the HW calculated using Eq. (2). The results are gathered for gold slits and ridges with widths $0.27 \lambda$ and ridge height $0.27 \lambda$. From bottom to top, the illuminations correspond to a plane wave, the fundamental slit $\mathrm{TEM}_{00}$ mode, a HW generated by a $y$-polarized magnetic line source located on the surface at $x=-\lambda$, and a SPP mode. The dashed and solid arrows on the surface represent HWs and SPPs, respectively, the arrows in the slit represent fundamental slit modes, and other arrows in free space represent plane waves. The arrows denoting incident and scattered waves are in red and in green, respectively. This notation of arrows is consistently used in the following figures.

larization (magnetic vector along the invariant $y$ direction). Since the field generated on the surface is responsible for the electromagnetic interaction between nearby indentations, it is carefully examined in Fig. 1 (black solid curves) for various incident illuminations and various slit and ridge geometries. The calculation is performed using an aperiodic-Fourier modal method (a-FMM), ${ }^{28,29}$ a fully vectorial frequencydomain modal method. The a-FMM is a generalization of the well-developed rigorous coupled wave analysis (RCWA), ${ }^{30}$ and includes perfectly matched layers to handle the outgoing wave conditions. It is essential to keep in mind that, in Fig. 1 , the scattered fields have all been normalized such that $H_{y}(|x|=\lambda)=1$. Otherwise, their phases and amplitudes would strongly vary from one curve to the other. Intrinsically, what counts in the figure is the field shape, which is found to solely depend on the frequency and to be merely independent of the incident illumination and of the scatterer geometry. We emphasize that this result is not obtained for very tiny scatterers that operate in the weak scattering regime and that are well predicted by perturbation theory but for weakly sub- $\lambda$ indentations (slits or ridges with dimensions up to $\approx \lambda / 3$ ) that incorporate localized resonances and that are currently of great importance for plasmonic and metamaterial devices. ${ }^{1-12,17-19}$

This observation can be theoretically justified by the fact that, under illumination by an external TM-polarized electric field $\mathbf{E}^{\text {ext }}=E_{x}^{\text {ext }} \mathbf{x}+E_{z}^{\text {ext }} \mathbf{z}$, a small nonmagnetic $2 \mathrm{D}$ indentation (at $x=z=0$ ) can be approximately represented by two effective electric line sources (invariant in the $y$ direction), polarized either parallel or perpendicular to the interface $(z=0)$, $\mathbf{p}_{x}=\left(\sigma_{x x} E_{x}^{\mathrm{ext}}+\sigma_{x z} E_{z}^{\mathrm{ext}}\right) \mathbf{x}$, or $\mathbf{p}_{z}=\left(\sigma_{z x} E_{x}^{\mathrm{ext}}+\sigma_{z z} E_{z}^{\mathrm{ext}}\right) \mathbf{z}$, where $\boldsymbol{\sigma}$ is a $2 \times 2$ polarizability tensor. Recently we analytically solved the problem of light emission by an oscillating electric line source on a metal-dielectric interface, ${ }^{25}$ and provided that $\left|\varepsilon_{m}\right| \gg \varepsilon_{d}$ (a condition readily met in practice for metals illuminated below their plasma frequencies), we found that the electromagnetic fields emitted by the $x$ - and $z$-polarized electric line sources are the same, and are also equal to the ra- diation by a $y$-polarized magnetic line source. In other words, the three Green's-function-tensor source components degenerate and only differ by a complex proportionality factor. Therefore, even if the polarizability tensor of sub- $\lambda$ indentations strongly depends on the incident illumination and on the actual indentation geometry, size, and refractive index, the overall shape (not the complex amplitude) of the field scattered on every side of an arbitrary sub- $\lambda$ indentation illuminated by an arbitrary incident field may be regarded as a fixed quantity. The latter is nothing else than the HW previously introduced and is approximately proportional to the field response at $(x, z)$ to a $y$-polarized magnetic line source at $x=z=0$ on the metal surface. Denoting by $H_{\mathrm{HW}}^{+}$and $H_{\mathrm{HW}}^{-}$the scattered HWs launched on every side of the indentation, we may write

$$
H_{y}(x, z)=c^{+} H_{\mathrm{HW}}^{+}(x, z)+c^{-} H_{\mathrm{HW}}^{-}(x, z),
$$

where $c^{+}$and $c^{-}$are complex excitation coefficients (see the inset of Fig. 1), and as shown in several recent reports, ${ }^{22-25}$ $H_{\mathrm{HW}}^{+}$is given by

$$
\begin{aligned}
H_{\mathrm{HW}}^{+}(x, 0) & =H_{\mathrm{SP}}^{+}(x, 0)+H_{\mathrm{CW}}^{+}(x, 0) \\
& =\exp \left(i k_{\mathrm{SP}} x\right)+\left(2 \pi \frac{k_{\mathrm{SP}}^{2}}{k_{0}^{2}} \frac{\sqrt{\varepsilon_{d} \varepsilon_{m}}}{\varepsilon_{m}-\varepsilon_{d}}\right)^{-1}\left(I_{m}+I_{d}\right),
\end{aligned}
$$

for $x>0$ and 0 otherwise. The HW is composed of a fixed mixing ratio $^{31}$ between an SPP contribution, $H_{\mathrm{SP}}^{+}(x, 0)$ $=\exp \left(i k_{\mathrm{SP}} x\right)$, and a quasi-CW contribution, $H_{\mathrm{CW}}^{+}(x, 0)$. In Eq. (2), $k_{\mathrm{SP}}=k_{0}\left[\varepsilon_{d} \varepsilon_{m} /\left(\varepsilon_{d}+\varepsilon_{m}\right)\right]^{1 / 2}$ is the in-plane SPP propagation constant $\left(k_{0}=2 \pi / \lambda\right.$ being real $)$ and $I_{m}=\exp$ $(-i \pi / 4) \frac{\varepsilon_{d}}{\varepsilon_{m}-\varepsilon_{d}} \int_{0}^{+\infty} \frac{\exp \left(i k_{0} x \sqrt{\varepsilon_{m}+i t}\right) \sqrt{t}}{\left[1-\left(\varepsilon_{m}+i t\right) k_{0}^{2} / k_{\mathrm{SP}}^{2}\right] \sqrt{\varepsilon_{m}+i t}} d t, I_{d}$ being obtained from $I_{m}$ by exchanging $\varepsilon_{d}$ and $\varepsilon_{m} . I_{m}+I_{d}$ can be calculated by numerical integration and asymptotically behaves as $x^{-1 / 2} \exp \left(i k_{0} \varepsilon_{d}^{1 / 2} x\right)$ in the vicinity of the scatterer. ${ }^{22-25}$ Note that due to symmetry reasons, $H_{\mathrm{HW}}^{-}(x, z)=H_{\mathrm{HW}}^{+}(-x, z)$. 


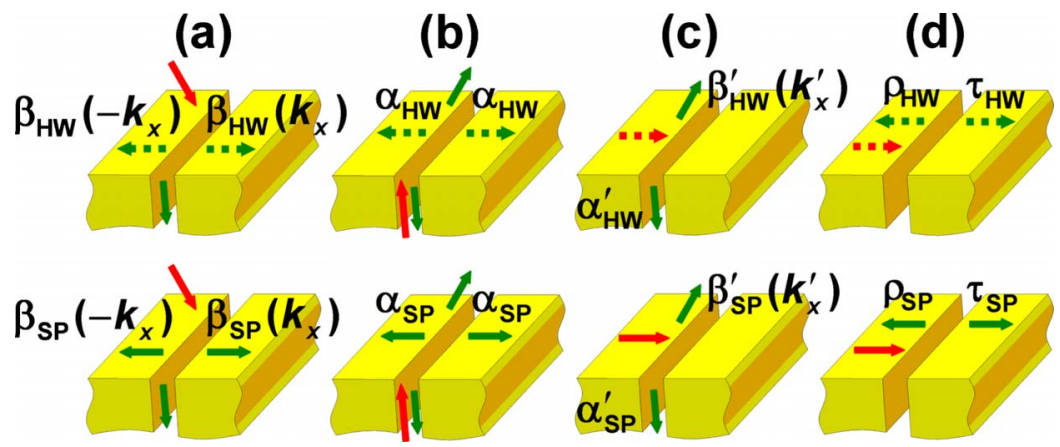

FIG. 2. (Color online) Elementary scattering events of HW (upper row) and of SPP (lower row) for a centrosymmetric slit. [(a) and (b)] Scattering coefficients $\beta_{\mathrm{HW}}\left(k_{x}\right)$ or $\alpha_{\mathrm{HW}}$ [respectively, $\beta_{\mathrm{SP}}\left(k_{x}\right)$ or $\alpha_{\mathrm{SP}}$ ] corresponding to HW (respectively, SPP) excitations under illumination either by a TM-polarized incident plane wave with an in-plane parallel wave vector $k_{x}$ or by the fundamental slit mode. (c) Reciprocal scattering coefficients $\beta_{\mathrm{HW}}^{\prime}\left(k_{x}^{\prime}\right)$ and $\alpha_{\mathrm{HW}}^{\prime}$ [respectively, $\beta_{\mathrm{SP}}^{\prime}\left(k_{x}^{\prime}\right)$ and $\alpha_{\mathrm{SP}}^{\prime}$ ] under HW (respectively, SPP) illumination, where $k_{x}^{\prime}$ denotes in-plane parallel wave vectors of scattered plane waves. (d) In-plane scattering coefficients $\tau_{\mathrm{HW}}$ and $\rho_{\mathrm{HW}}$ (respectively, $\tau_{\mathrm{SP}}$ and $\left.\rho_{\mathrm{SP}}\right)$ that characterize the transmission and the reflection of HWs (respectively, SPPs).

As shown in Fig. 1, the HW expression of Eq. (2) (red dashed curves) is a very good approximation for the actual fields (black solid curves) scattered by sub- $\lambda$ indentations with finite dimensions up to $\approx \lambda / 3$. We have performed other calculations for other wavelengths down to $\lambda=500 \mathrm{~nm}$, for other geometries such as grooves, hole chains (see Sec. IV), or noncentrosymmetric indentations, and for various transversal sizes of sub- $\lambda$ indentations. We always found that the overall shape of the scattered field weakly depends on the incident field and on the actual details of the sub- $\lambda$ scatterer, and that the HW expression of Eq. (2) is a trusty approximation of the scattered field, which is rather robust to the indentation size; only the excitation coefficients (the $c^{+}$and $c^{-}$) strongly depend on the incident illumination and on the fine structure of the indentation (just like the polarizability tensor $\boldsymbol{\sigma})$.

\section{DEFINITION AND CALCULATION OF SCATTERING COEFFICIENTS FOR HYBRID WAVES}

The previous analysis sets the HW at the heart of the scattering physics of sub- $\lambda$ metallic surfaces: since external fields illuminating the surface locally excite HWs [Figs. 2(a) and 2(b)] and since the launched HWs further scatter into new HWs [Fig. 2(d)], we anticipate that the near-field response of arbitrary sub- $\lambda$ metallic surfaces can be modeled as a pure superposition of HWs, see Eq. (4) hereafter. A crucial point is how to determine the HW superposition?

\section{A. Definition of HW scattering coefficients}

In Sec. IV, the HW superposition is determined through a coupled-HW model that relies on HW scattering coefficients associated with every individual indentation of the surface (upper row of Fig. 2). The model fully preserves and further generalizes the intuitive picture of a wave progression through multiple scattering on the surface, which constitutes the main strength of classical SPP models. ${ }^{4-16}$ For instance, the HW-to-HW scattering [upper row of Fig. 2(d)] contains not only the classical SPP-to-SPP scattering [lower row of Fig. 2(d), Refs. 4-16] but also the conversion of quasi-CW into SPPs (Ref. 32) by scattering on a sub- $\lambda$ defect.

However, scattering coefficients are only defined for normal modes ${ }^{33}$ in classical theories. ${ }^{34,35}$ Because HWs are not normal modes (they are a superposition of modes), they do not obey orthogonality or reciprocity relationships. ${ }^{34,35}$ Thus one may think that it is difficult (or even impossible) to define scattering coefficients for those waves. In the following, we overcome this difficulty and show that, not only scattering coefficients can be defined for HWs but also they can be approximately calculated as "classical" SPP scattering coefficients for any geometry and for any frequency.

The SPP scattering coefficients corresponding to the HW ones are shown in the lower row of Fig. 2. The subscripts "SP" or "HW" are related to SPP or HW quantities, respectively. To establish the correspondence between SPP and HW scattering coefficients, let us start by considering the launching of HWs or SPPs under the illumination by a plane wave or by a slit mode. Since the HW is a combination of SPP and quasi-CW waves with a fixed mixing ratio ${ }^{31}$ at a given wavelength, the HW excitation rates may be directly derived from SPP excitation rates, and we have [Figs. 2(a) and 2(b)]

$$
\beta_{\mathrm{HW}}\left(k_{x}\right) \approx \beta_{\mathrm{SP}}\left(k_{x}\right), \quad \alpha_{\mathrm{HW}} \approx \alpha_{\mathrm{SP}},
$$

where $k_{x}$ denotes the in-plane parallel wave vector of the incident plane wave. Then by noting that, although different by nature, the HW and SPP fields share many electromagnetic properties close to the metal surface (almost identical propagation constants, similar penetration depth in the metal...), one may invoke a form of causality principle, to further infer that the fields scattered by any indentation illuminated by either a HW or a SPP are nearly identical. ${ }^{36}$ Using the same HW and SPP normalizations, this implies that [Figs. 2(c) and 2(d)]

$$
\begin{gathered}
\beta_{\mathrm{HW}}^{\prime}\left(k_{x}^{\prime}\right) \approx \beta_{\mathrm{SP}}^{\prime}\left(k_{x}^{\prime}\right), \quad \alpha_{\mathrm{HW}}^{\prime} \approx \alpha_{\mathrm{SP}}^{\prime}, \quad \rho_{\mathrm{HW}} \approx \rho_{\mathrm{SP}}, \\
\tau_{\mathrm{HW}} \approx \tau_{\mathrm{SP}}-1,
\end{gathered}
$$

where $k_{x}^{\prime}$ represents the in-plane parallel wave vectors of the scattered plane waves. The minus-one term in the last equality comes from the fact that the amount of SPPs scattered in 
transmission is the difference between the total SPP field (with an amplitude $\tau_{\mathrm{SP}}$ ) and the incident SPP (with unitary amplitude).

Equations (3a) and (3b) are remarkably simple and readily relate nonintuitive $H W$ scattering coefficients to classical SPP ones. It follows that, although the HW-multiplescattering model is much more accurate than the classical pure-SPP model (as shown in Sec. IV), its implementation does not require additional computation loads; like the SPP model, it relies on the sole knowledge of the SPP scattering coefficients of every indentation.

\section{B. Numerical calculation of HW scattering coefficients}

To calculate the HW scattering coefficients, one may consider a given indentation illuminated by a given incident field, either a plane wave, a slit mode, or a HW..., and solves the scattering problem with a fully vectorial numerical method. A HW incident field can be generated by a magnetic line source located on the surface in the vicinity of the indentation. Then one may extract the coefficient of the HW that forms the scattered field, by considering the field scattered on the surface for instance. Although this approach is valid, we rather prefer taking benefit from the correspondence between SPP and HW scattering coefficients. Indeed, this is more convenient simply because SPP scattering coefficients are well-defined modal quantities. Furthermore, there are several advantages: (1) the SPP scattering coefficients can be easily calculated with various numerical tools. (2) Their computation loads can be lowered by considering reciprocity relationships. $^{34,35}$ (3) More importantly, SPP scattering coefficients are physical and intuitive quantities, 32,37 which are at the heart of the physics of sub- $\lambda$ metallic surfaces, even at far-infrared wavelengths for which SPPs negligibly contribute to HWs that are mainly formed by quasiCWs; we believe that it is worth gathering information on these coefficients to help further designs.

To calculate the SPP scattering coefficients, we use the fully vectorial a-FMM. ${ }^{28,29}$ More specifically, referring to the lower row of Fig. 2(a) or Fig. 2(b), the calculation of $\beta_{\mathrm{SP}}\left(k_{x}\right)$ or $\alpha_{\mathrm{SP}}$ relies on the solution of the scattering problem for the isolated indentation under illumination by a plane wave or by the fundamental slit mode. Then $\beta_{\mathrm{SP}}\left(k_{x}\right)$ or $\alpha_{\mathrm{SP}}$ is obtained by calculating an overlap integral between the scattered field and the analytically known SPP field. Details about the integral are given in Ref. 38 . The calculation of $\beta_{\mathrm{SP}}^{\prime}\left(k_{x}^{\prime}\right), \alpha_{\mathrm{SP}}^{\prime}, \tau_{\mathrm{SP}}$, and $\rho_{\mathrm{SP}}$ is associated with the scattering problem shown in the lower row of Figs. 2(c) or Fig. 2(d), where the indentation is illuminated by a SPP. $\tau_{\mathrm{SP}}$ and $\rho_{\mathrm{SP}}$ are readily obtained as modal coefficients of the scattering matrix, ${ }^{39} \beta_{\mathrm{SP}}^{\prime}\left(k_{x}^{\prime}\right)$ is obtained by performing a plane-wave decomposition ${ }^{40}$ of the scattered field, and $\alpha_{\mathrm{SP}}^{\prime}$ can be extracted from the total field by using the orthogonality relationship of slit modes. ${ }^{34,35} \mathrm{By}$ virtue of the Lorentz reciprocity theorem ${ }^{34,35}$ of normal modes, $\beta_{\mathrm{SP}}\left(k_{x}\right)$ can be calculated in a more efficient way by using $^{37} \beta_{\mathrm{SP}}\left(k_{x}\right)=\beta_{\mathrm{SP}}^{\prime}\left(k_{x}\right)$, similarly, $\alpha_{\mathrm{SP}}^{\prime}=\alpha_{\mathrm{SP}}$. For noncentrosymmetric indentations, above discussions on the definition and calculation of HW scattering coefficients can be generalized without difficulty (see Appendix A).
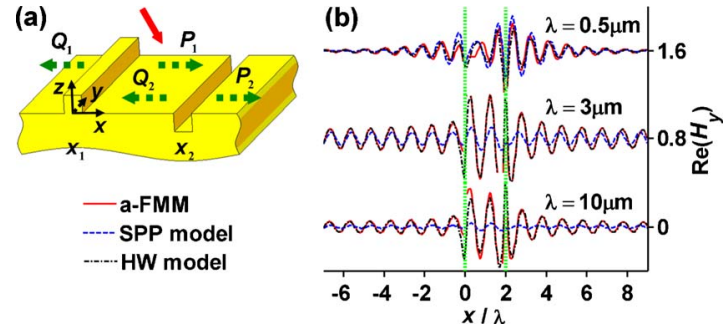

FIG. 3. (Color online) Test of the HW model for the gold ridgegroove doublet shown in (a). The doublet distance is $d=2 \lambda$. The ridge and groove widths and heights are $w=h=0.1 \lambda$. (b) Magnetic field scattered on the surface $(z=0)$ for several wavelengths $(\lambda$ $=0.5,3$, and $10 \mu \mathrm{m})$. The predictions of the HW model [Eqs. (4) and (5)] and of the classical SPP model are shown with black dashed-dotted and blue dashed curves; fully vectorial a-FMM computational results are shown with the red solid curves. The vertical green dotted lines show the ridge and groove boundaries. The data are obtained for a normally incident plane wave $\left(H_{y}=1\right.$ at $\left.z=0\right)$, similar results being obtained for oblique incidence.

\section{HYBRID-WAVE MULTIPLE-SCATTERING MODEL}

Developing the HW multiple-scattering model for the general case of a surface with $N$ arbitrary indentations is postponed to Appendix A. For the sake of illustration, it is sufficient to restrict ourselves to the ridge-groove doublet shown in Fig. 3(a). This allows us to present the model with an example that is simple, albeit interesting, since it combines different sub- $\lambda$ indentations. The scattered magnetic field $H_{y}(x, z)$ of the two-body system is composed of four HWs,

$$
H_{y}(x, z)=\sum_{n=1,2}\left[P_{n} H_{\mathrm{HW}}^{+}\left(x-x_{n}, z\right)+Q_{n} H_{\mathrm{HW}}^{-}\left(x-x_{n}, z\right)\right],
$$

where $x_{1}$ and $x_{2}$ denote the ridge and groove locations, and $P_{n}$ and $Q_{n}(n=1,2)$ represent the unknown coefficients of the two HWs that are launched at the $n$th indentation and that propagate in the positive and negative $x$ directions. The set of coupled-HW equations is given by

$$
\begin{gathered}
P_{1}=W_{1} \beta_{\mathrm{HW}, 1}\left(k_{x}\right)+H_{\mathrm{HW}}^{-}\left(x_{1}-x_{2}, 0\right) \rho_{\mathrm{HW}, 1} Q_{2}, \\
P_{2}=W_{2} \beta_{\mathrm{HW}, 2}\left(k_{x}\right)+H_{\mathrm{HW}}^{+}\left(x_{2}-x_{1}, 0\right) \tau_{\mathrm{HW}, 2} P_{1}, \\
Q_{1}=W_{1} \beta_{\mathrm{HW}, 1}\left(-k_{x}\right)+H_{\mathrm{HW}}^{-}\left(x_{1}-x_{2}, 0\right) \tau_{\mathrm{HW}, 1} Q_{2}, \\
Q_{2}=W_{2} \beta_{\mathrm{HW}, 2}\left(-k_{x}\right)+H_{\mathrm{HW}}^{+}\left(x_{2}-x_{1}, 0\right) \rho_{\mathrm{HW}, 2} P_{1},
\end{gathered}
$$

where $\beta_{\mathrm{HW}, n}, \tau_{\mathrm{HW}, n}$, and $\rho_{\mathrm{HW}, n}$ are the HW scattering coefficients defined in Fig. 2 for the $n$th indentation, and $W_{n}$ $=\exp \left(i k_{x} x_{n}\right)$ is the in-plane phase delay of the TM-polarized incident plane wave at the $n$th indentation. Equation (5) are intuitive. Referring to Eq. (5a) for instance, the total HW launched by the ridge in the positive $x$ direction is seen to result from two contributions, a first one $\left(\beta_{\mathrm{HW}, 1}\right)$ occasioned by the incident plane wave and a second one due to the scattering $\left(\rho_{\mathrm{HW}, 1}\right)$ of a damped $\left[H_{\mathrm{HW}}^{-}\left(x_{1}-x_{2}, 0\right)\right] \mathrm{HW}$ initially launched at the groove with an amplitude $Q_{2}$. 


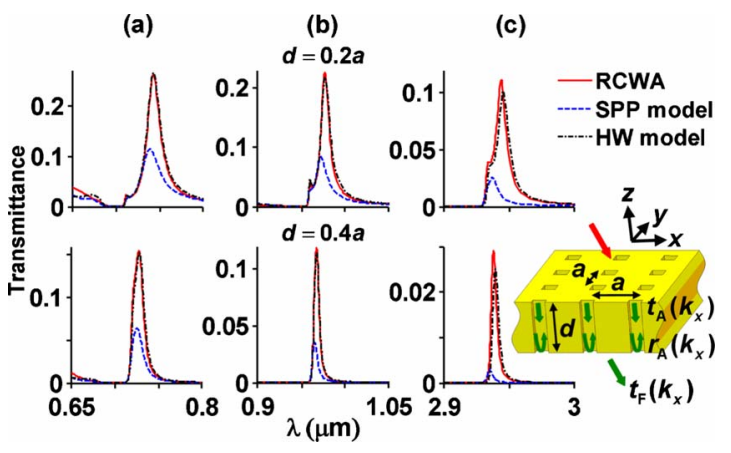

FIG. 4. (Color online) Test of the HW model for the EOT. The fully vectorial RCWA computational results and the SPP-model predictions for the transmittance are shown with red solid and blue dashed curves. The predictions of the HW model are shown with black dashed-dotted curves; they are almost superimposed with the RCWA results. The data are collected for a gold membrane in air perforated by a $2 \mathrm{D}$ array of square holes (inset) illuminated by a normally incident $\left(k_{x}=0\right)$ plane wave. The hole side length is $0.28 a$ and the membrane thickness is $d=0.2 a$ (upper row) or $0.4 a$ (lower row), $a$ being the grating pitch. Three spectral bands are covered, from visible to near-infrared frequencies: (a) $a=0.68 \mu \mathrm{m}$, (b) $0.94 \mu \mathrm{m}$, and (c) $2.92 \mu \mathrm{m}$.

The practical implementation of the HW model is twofold. Using fully vectorial modal methods, we first calculate the SPP scattering coefficients of the ridge and of the groove and further identify them as the HW scattering coefficients $\beta_{\mathrm{HW}, n}, \tau_{\mathrm{HW}, n}$, and $\rho_{\mathrm{HW}, n}$. We then straightforwardly solve linear Eqs. (5a)-(5d) for the unknown coefficients $P_{n}$ and $Q_{n}$.

As shown in Fig. 3(b), the model predictions are highly accurate from green light $(\lambda=0.5 \mu \mathrm{m})$ just above the gold plasma wavelength to thermal wavelengths $(\lambda=10 \mu \mathrm{m})$, and beyond as shown by other computational results not reported here for compactness. In comparison, classical SPP models that neglect quasi-CWs are only approximately valid at small wavelengths, and rapidly become inaccurate as one considers longer wavelengths, where quasi-CWs are the dominant contribution to HWs. ${ }^{22-25}$ The results are obtained for indentation sizes of $0.1 \lambda$ (this already covers many geometries of current interest $\left.1^{1-3,17-19}\right)$. A similar accuracy is obtained for larger indentations, $w=h=0.2 \lambda$, and with a slight modification, indentation sizes as large as $0.6 \lambda$ may be handled with a high accuracy (see Appendix B).

The high accuracy achieved for the near-field data in Fig. 3 (b) is not fortuitous. We have additionally tested the model for far-field data obtained for the extraordinary optical transmission (EOT) (Ref. 41) through a 2D fully periodic sub- $\lambda$ hole array (period $a$ ) drilled in a noble-metal membrane in air (inset of Fig. 4). Besides that the EOT represents a difficult scattering problem, this test is motivated by the fact that, since a pure-SPP model ${ }^{14}$ has already been elaborated for this phenomenon, one may quantify the net benefit of the present generalized theory.

The hole array is a geometry that is much more complex than the ridge-groove doublet, as it incorporates two interfaces, a coupling of HWs to evanescent fields in the holes, three-dimensional (3D) indentations rather than 2D ones... Under the assumption that the energy transfer through the metallic membrane is mediated by the fundamental Bloch mode of the hole array, ${ }^{14,42}$ the zeroth-order transmission coefficient $t_{\mathrm{F}}\left(k_{x}\right)$ can be expressed as a classical Fabry-Perot equation,

$$
t_{\mathrm{F}}\left(k_{x}\right)=\frac{t_{\mathrm{A}}^{2}\left(k_{x}\right) \exp \left(i k_{0} n d\right)}{1-r_{\mathrm{A}}^{2}\left(k_{x}\right) \exp \left(i 2 k_{0} n d\right)},
$$

where $t_{\mathrm{A}}\left(k_{x}\right), r_{\mathrm{A}}\left(k_{x}\right)$, and $n$ are, respectively, the transmission coefficient, the reflection coefficient, and the complex effective index of the fundamental Bloch mode of the hole array (see the inset of Fig. 4), $k_{x}$ being the in-plane $x$ component of the wave vector of the obliquely incident plane wave under TM polarization (magnetic vector parallel to $y$ axis), and $d$ is the thickness of the metal membrane.

The general HW-multiple-scattering model developed in Appendix A can be directly applied for deriving analytical expressions of $t_{\mathrm{A}}\left(k_{x}\right)$ and $r_{\mathrm{A}}\left(k_{x}\right)$ by considering the $y$-periodic one-dimensional hole chain as the elementary indentation. For a periodic array of identical indentations, $W_{n}$ $=w^{n}=\exp \left(i k_{x} n a\right)$ and the pseudoperiodic (Floquet) boundary conditions $\left(P_{n}=w P_{n-1}, Q_{n}=w Q_{n-1}\right)$ drastically simplify the analysis; the system of linear Eq. (A2) can be solved analytically. For normal incidence $\left(k_{x}=0\right)$, we get

$$
\begin{gathered}
t_{\mathrm{A}}=t+\frac{2 \alpha_{\mathrm{SP}} \beta_{\mathrm{SP}}}{\left(1 / \Sigma H_{\mathrm{HW}}+1\right)-\left(\rho_{\mathrm{SP}}+\tau_{\mathrm{SP}}\right)}, \\
r_{\mathrm{A}}=r+\frac{2 \alpha_{\mathrm{SP}}^{2}}{\left(1 / \Sigma H_{\mathrm{HW}}+1\right)-\left(\rho_{\mathrm{SP}}+\tau_{\mathrm{SP}}\right)},
\end{gathered}
$$

where all the HW scattering coefficients have been replaced by the SPP ones according to Eq. (3). The scattering coefficients $\alpha_{\mathrm{SP}}, \beta_{\mathrm{SP}}=\beta_{\mathrm{SP}}\left(k_{x}=0\right), \rho_{\mathrm{SP}}, \tau_{\mathrm{SP}}, r$, and $t=t\left(k_{x}=0\right)$ have been defined and calculated in Ref. 14. In Eq. (7), $\Sigma H_{\mathrm{HW}}$ $=\sum_{n=1}^{\infty} H_{\mathrm{HW}}^{+}(n a, 0)=\sum_{n=1}^{\infty} H_{\mathrm{CW}}^{+}(n a, 0)+\left[\exp \left(-i k_{\mathrm{SP}} a\right)-1\right]^{-1}$, which represents a field summation over all the individual HWs scattered by every individual chain. Actually, Eq. (7) are almost identical to those obtained with the classical pure-SPP model [Eqs. (1) and (2) in Ref. 14]. The only difference is the apparition of a new term in the resonant denominator, $\sum_{n=1}^{\infty} H_{\mathrm{CW}}^{+}(n a, 0)$, which corresponds to the contribution of all the individual quasi-CWs scattered by the chains of the array. Since this term can be easily calculated numerically, we conclude that the additional efforts required to incorporate the quasi-CW into the classical pure-SPP model is negligible.

The main predictions of the HW model for the zerothorder transmittance $\left|t_{\mathrm{F}}\right|^{2}$ are shown in Fig. 4 and are compared with those of the pure-SPP model and with RCWA data. It is noticeable that despite the complexity of the holearray geometry, the deviation of the HW-model predictions from the RCWA data is imperceptible at the scale of the figures, and this for the three spectral bands covering visible and infrared radiations. The present model well explains why the EOT phenomenon exists at far-infrared frequencies and even for perfect conductors, ${ }^{43}$ in a regime where SPPs are very weakly excited and classical SPP models completely fail at predicting any transmission. It additionally offers an insight into one of the major initial topics in grating theory, 


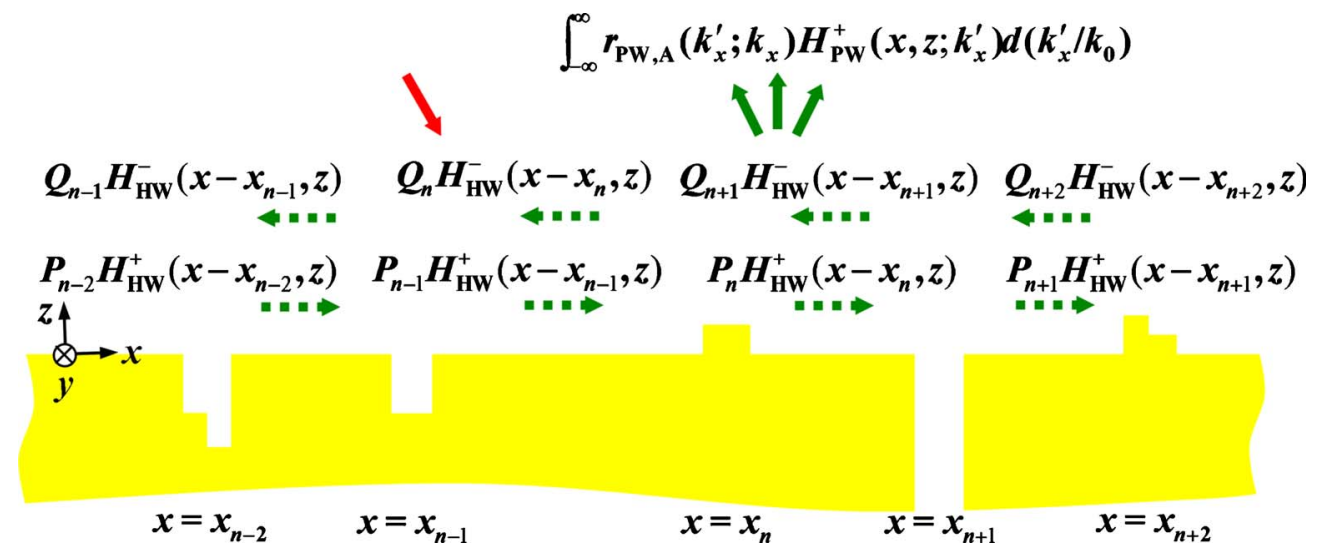

FIG. 5. (Color online) Definition of wave coefficients for a general sub- $\lambda$ metallic surface composed of $N$ 2D indentations that are possibly different. The surface is illuminated by a TM-polarized (magnetic vector parallel to $y$-axis) plane wave impinging at oblique incidence. $P_{n}$ and $Q_{n}$, respectively, denote the coefficients of the right- and left-going HWs [magnetic field denoted by $H_{\mathrm{HW}}^{+}\left(x-x_{n}, z\right)$ and $H_{\mathrm{HW}}^{-}\left(x-x_{n}, z\right)$, respectively], which originate from the $n$th indentation located at $x=x_{n}(n=1, \ldots, N)$.

and may be considered as a structuring of Fano's intuitive explanation of Wood's anomaly. ${ }^{20,21}$ Such resonances can also be obtained by computing the complex zeros of the denominator in Eq. (7). Such poles correspond to the eigenmodes of the periodically perforated metallic surface. ${ }^{42} \mathrm{Fi}-$ nally, let us note that since the HW model is accurate, it may be used with confidence to design EOT-related devices over the entire electromagnetic spectrum below the metal plasma frequency. A quantitative analysis of the physics of Eq. (7) is out of the scope of the present report and more details will be published in another paper.

\section{SUMMARY}

We have proposed an intuitive model of light scattering on sub- $\lambda$ metallic surfaces. The model relies on the fundamental hypothesis that the near field of the sub- $\lambda$ metallic surfaces is composed of a superposition of the same identical entity even if the indentations of the sub- $\lambda$ surfaces are not all identical and even if the indentation sizes are as large as $0.2 \lambda$. A priori, the sub- $\lambda$ indentations can be any type of $2 \mathrm{D}$ structures; we have checked the hypothesis for metallic ridges, for grooves, and for asymmetric grooves but we believe that it should also remain valid for other indentations, such as subsurface structures for instance. We have called the generic entity a hybrid wave to emphasize that it is a mix of SPP and quasi-CW waves. We have further defined scattering coefficients for these waves, and we have been able to derive a multiple-scattering formalism, which describes how these waves propagate and scatter on the surface.

On overall, the electromagnetic property of complex metallic surfaces composed of different sub- $\lambda$ indentations placed on the surface at arbitrary positions can just be analyzed from the knowledge of how every indentation scatters the incident HW. The model not only retains all the intuitive and helpful features of classical SPP models but also provides highly accurate predictions. We expect that the present model, which provides a comprehensive picture of sub- $\lambda$ metallic surfaces from visible to microwave frequencies, may help further understanding and engineering the optical prop- erties of plasmonic surfaces and of metamaterials. In this context, it would be important to generalize the HW concept to point-defect local scatterers, ${ }^{44,45}$ and thus to enable a microscopic treatment of arbitrary $3 \mathrm{D}$ sub- $\lambda$ metallic surfaces.

\section{ACKNOWLEDGMENTS}

The authors thank J. P. Hugonin for fruitful discussions. H.T.L. acknowledges financial supports from the National Natural Science Foundation of China (Grant No. 10804057), from the Cultivation Fund of the Key Scientific and Technical Innovation Project, Ministry of Education of China (Grant No. 708021), from the Program for New Century Excellent Talents in University (Program No. NCET-08-0289), and from the 973 Project (Project No. 2007CB307001). Correspondence and requests for materials should be addressed to Haitao Liu (liuht@nankai.edu.cn).

\section{APPENDIX A: GENERAL HW-MULTIPLE-SCATTERING MODEL}

Let us consider the general case of a sub- $\lambda$ metallic surface composed of an arbitrary set of $N$ potentially different 2D indentations located at arbitrary positions on the surface. The surface is assumed to be illuminated by a TM-polarized plane wave (Fig. 5).

The derivation starts with the premise that the near field of the metal surface can be modeled as a superposition of $H W s$ that are launched by every indentation. This is a direct consequence of our finding that the field scattered by any sub- $\lambda$ indentation is approximately a HW, whatever the illumination is. Thus under illumination by an incident plane wave, the indentations generate HWs, which further generate HWs again by scattering with nearby indentations. All things considered, only $H W$ s are at work on the $s u b-\lambda$ surface.

The procedure is twofold. After writing and solving a set of self-consistent coupled-HW equations, the unknown coefficients of the HWs scattered by every indentation are first determined. This first step determines the near field in the vicinity of the surface. Then the plane-wave continuum scat- 
tered in the far field is calculated through a summation over all radiated waves that are scattered by every indentation.

Near field. As sketched in Fig. 5, we use $P_{n}$ and $Q_{n}$ to denote the unknown coefficients of the HWs that are scattered by the $n$th indentation at $x=x_{n}(n=1, \ldots, N)$ and that propagate toward the positive and negative $x$ directions, respectively. The scattered field can thus be expressed as a superposition of $2 N \mathrm{HWs}$,

$$
H_{y}(x, z)=\sum_{n=1}^{N}\left[P_{n} H_{\mathrm{HW}}^{+}\left(x-x_{n}, z\right)+Q_{n} H_{\mathrm{HW}}^{-}\left(x-x_{n}, z\right)\right],
$$

where $H_{\mathrm{HW}}^{+}(x, z)$ and $H_{\mathrm{HW}}^{-}(x, z)$ are defined in Eq. (1). Referring to the HW scattering coefficients defined in Fig. 2, a set of $2 N$ coupled-HW equations with $2 N$ unknowns (the $P_{n}$ and $Q_{n}$ ) is straightforwardly written as

$$
\begin{aligned}
P_{n}= & W_{n} \beta_{\mathrm{HW}, n}^{+}\left(k_{x}\right)+\tau_{\mathrm{HW}, n}^{+} \sum_{m=0}^{n-1} P_{m} H_{\mathrm{HW}}^{+}\left(x_{n}-x_{m}, 0\right) \\
& +\rho_{\mathrm{HW}, n}^{-} \sum_{m=n+1}^{N+1} Q_{m} H_{\mathrm{HW}}^{-}\left(x_{n}-x_{m}, 0\right), \\
Q_{n}= & W_{n} \beta_{\mathrm{HW}, n}^{-}\left(k_{x}\right)+\rho_{\mathrm{HW}, n}^{+} \sum_{m=0}^{n-1} P_{m} H_{\mathrm{HW}}^{+}\left(x_{n}-x_{m}, 0\right) \\
& +\tau_{\mathrm{HW}, n}^{-} \sum_{m=n+1}^{N+1} Q_{m} H_{\mathrm{HW}}^{-}\left(x_{n}-x_{m}, 0\right),
\end{aligned}
$$

where $n=1, \ldots, N, W_{n}=\exp \left(i k_{x} x_{n}\right)$ is the in-plane phase delay of the incident plane wave at the $n$th indentation, and $\rho_{\mathrm{HW}, n}^{ \pm}, \tau_{\mathrm{HW}, n}^{ \pm}$, and $\beta_{\mathrm{HW}, n}^{ \pm}\left(k_{x}\right)$ are the HW scattering coefficients defined in Fig. 2 for the $n$th indentation. Here we use the additional "plus" and "minus" superscripts to discriminate HW scattering coefficients corresponding to right- or left-going electromagnetic waves for possibly noncentrosymmetric indentations. We additionally have $P_{0}=Q_{N+1}=0$ to specify that no HW impinges from the two outer sides of the indentation ensemble. Equation (A2) describe the scattering processes that occur at the $n$th indentation. They can be understood intuitively. Referring to Eq. (A2a) for instance, the first term represents the right-going $\mathrm{HW}$ launched by the incident plane wave; the second term represents a sum over all the right-going HWs that are excited by all the individual right-going HWs, the latter being generated at indentations located on the left side of the $n$th indentation $(m<n)$ and further propagating $\left[H_{\mathrm{HW}}^{+}\left(x_{n}-x_{m}, 0\right)\right]$ to the $n$th indentation before being scattered $\left(\tau_{\mathrm{HW}, n}^{+}\right)$there. The third term has a similar interpretation, and represents a sum over all the rightgoing HWs at the $n$th indentation excited by all the leftgoing HWs that are generated at indentations located on the right side of the $n$th indentation $(m>n)$. Equation (A2b) represents the excitation of the left-going HWs and can be understood in a similar way. Once all scattering coefficients $\rho_{\mathrm{HW}, n}^{ \pm}, \tau_{\mathrm{HW}, n}^{ \pm}$, and $\beta_{\mathrm{HW}, n}^{ \pm}\left(k_{x}\right)$ are calculated for every indentation, the set of $2 N$ linear equations defined by Eqs. (A2a) and (A2b) with $2 N$ unknowns, the $P_{n}$ and $\mathrm{Q}_{n}$, is easily solved with a matrix inversion. The associated computation loads are much weaker than those related to the fully vectorial calculations of the HW scattering coefficients (see Sec. III for details). Finally note that Eqs. (4) and (5) obtained for a ridge-groove geometry represent the special case $N=2$. The knowledge of the $P_{n}$ and $\mathrm{Q}_{n}$ entirely determines the near field of the sub- $\lambda$ surface. For the classical pure-SPP model, ${ }^{4-12}$ its equations can be directly obtained from Eqs. (A1) by removing the quasi-CW contribution (replacing $H_{\mathrm{HW}}^{ \pm}$ by $H_{\mathrm{SP}}^{ \pm}$and replacing the HW scattering coefficients by the corresponding SPP ones).

Far field. Once the HW coefficients $P_{n}$ and $\mathrm{Q}_{n}$ are known, in a second step, one may determine the electromagnetic field scattered in the far field. The latter can be expressed as a plane-wave superposition, and we have for the magnetic field

$$
H_{y}(x, z)=\int_{-\infty}^{\infty} r_{\mathrm{PW}, \mathrm{A}}\left(k_{x}^{\prime} ; k_{x}\right) H_{\mathrm{PW}}^{+}\left(x, z ; k_{x}^{\prime}\right) d\left(k_{x}^{\prime} / k_{0}\right),
$$

where $r_{\mathrm{PW}, \mathrm{A}}\left(k_{x}^{\prime} ; k_{x}\right)$ represents the coefficient of the planewave continuum that is scattered by all indentations and $H_{\mathrm{PW}}^{+}\left(x, z ; k_{x}^{\prime}\right)=\exp \left[i\left(k_{x}^{\prime} x+k_{z}^{\prime} z\right)\right]$ is the magnetic component of the scattered plane wave with an in-plane wave vector $k_{x}^{\prime}$. Let us now expand $r_{\mathrm{PW}, \mathrm{A}}\left(k_{x}^{\prime} ; k_{x}\right)$ as a sum over all the fields radiated at every indentation

$$
r_{\mathrm{PW}, \mathrm{A}}\left(k_{x}^{\prime} ; k_{x}\right)=\sum_{n=1}^{N} c_{\mathrm{PW}, n}\left(k_{x}^{\prime} ; k_{x}\right) / W_{n}^{\prime},
$$

where $c_{\mathrm{PW}, n}\left(k_{x}^{\prime} ; k_{x}\right)$ and $W_{n}^{\prime}=\exp \left(i k_{x}^{\prime} x_{n}\right)$ are respectively the coefficient and the in-plane phase delay of the plane wave that is scattered by the $n$th indentation. Again, we find three contributions for $c_{\mathrm{PW}, n}\left(k_{x}^{\prime} ; k_{x}\right)$,

$$
\begin{aligned}
c_{\mathrm{PW}, n}\left(k_{x}^{\prime} ; k_{x}\right)= & W_{n} r_{\mathrm{PW}, n}\left(k_{x}^{\prime} ; k_{x}\right)+\beta_{\mathrm{HW}, n}^{\prime+}\left(k_{x}^{\prime}\right) \sum_{m=0}^{n-1} P_{m} H_{\mathrm{HW}}^{+} \\
& \times\left(x_{n}-x_{m}, 0\right)+\beta_{\mathrm{HW}, n}^{\prime-}\left(k_{x}^{\prime}\right) \sum_{m=n+1}^{N+1} Q_{m} H_{\mathrm{HW}}^{-} \\
& \times\left(x_{n}-x_{m}, 0\right),
\end{aligned}
$$

where $\beta_{\mathrm{HW}, n}^{\prime \pm}\left(k_{x}^{\prime}\right)$ are defined in Fig. 2(c) for the $n$th indentation that is possibly noncentrosymmetric and $r_{\mathrm{PW}, n}\left(k_{x}^{\prime} ; k_{x}\right)$ is defined as a reflection coefficient from the incident plane wave to the plane-wave continuum back reflected by the $n$th indentation. The three terms in Eq. (A4b) represent the contributions from the incident plane wave, from all the rightgoing HWs and from all the left-going HWs, respectively.

Equations (A3), (A4a), and (A4b) provide an analytical expression for the electromagnetic field scattered in the far field, as the function of the near-field HW coefficients $P_{n}$ and $\mathrm{Q}_{n}$. Inserting Eq. (A4) into Eq. (A3) and using Eq. (A2) to express the $P_{n}$ and $\mathrm{Q}_{n}$, we finally get the total scattered far field in an explicit form, 


$$
\begin{aligned}
H_{y}(x, z)= & \sum_{n=1}^{N}\left\{W_{n} \int_{-\infty}^{\infty} r_{\mathrm{PW}, n}\left(k_{x}^{\prime} ; k_{x}\right) H_{\mathrm{PW}}^{+}\right. \\
& \times\left(x-x_{n}, z ; k_{x}^{\prime}\right) d\left(k_{x}^{\prime} / k_{0}\right) \\
& +\left[\sum_{m=0}^{n-1} P_{m} H_{\mathrm{HW}}^{+}\left(x_{n}-x_{m}, 0\right)\right] \int_{-\infty}^{\infty} \beta_{\mathrm{SP}, n}^{\prime+}\left(k_{x}^{\prime}\right) H_{\mathrm{PW}}^{+} \\
& \times\left(x-x_{n}, z ; k_{x}^{\prime}\right) d\left(k_{x}^{\prime} / k_{0}\right) \\
& +\left[\sum_{m=n+1}^{N+1} Q_{m} H_{\mathrm{HW}}^{-}\left(x_{n}-x_{m}, 0\right)\right] \\
& \left.\times \int_{-\infty}^{\infty} \beta_{\mathrm{SP}, n}^{\prime-}\left(k_{x}^{\prime}\right) H_{\mathrm{PW}}^{+}\left(x-x_{n}, z ; k_{x}^{\prime}\right) d\left(k_{x}^{\prime} / k_{0}\right)\right\},
\end{aligned}
$$

where the HW scattering coefficients, $\beta_{\mathrm{HW}, n}^{\prime \pm}\left(k_{x}^{\prime}\right)$, have been replaced by SPP ones, $\beta_{\mathrm{SP}, n}^{\prime \pm}\left(k_{x}^{\prime}\right)$, according to Eq. (3b). As shown in the lower row of Fig. 2, the three integrals in Eq. (A5) exactly correspond to the three far-field contributions due to the $n$th indentation under illumination by the incident plane wave [Fig. 2(a)], by the right-going SPP [Fig. 2(c)], and by the left-going SPP, respectively.

\section{APPENDIX B: REFINED HW MODEL FOR LARGE INDENTATIONS}

In Fig. 6, we show the influence of the indentation size on the model accuracy, for example, of the ridge-groove doublet [Fig. 3(a)]. As expected, the smaller the indentation, the better the model predictions. However, it is noticeable that the model remains very accurate even for indentations as large as $0.2 \lambda$. This already covers many geometries of current interest. ${ }^{1-3,17-19}$ As the indentation size increases further, the model accuracy gradually decreases, which indeed directly results from the Green's-function-tensor approximation for the HWs.

Actually, this limitation can be overcome by considering the actual field scattered by every indentation [its far-field part is expressed by Eq. (A5)], instead of its approximate expression (A1) in terms of HWs. For the ridge-groove doublet, the actual scattered field is composed of four contribu-

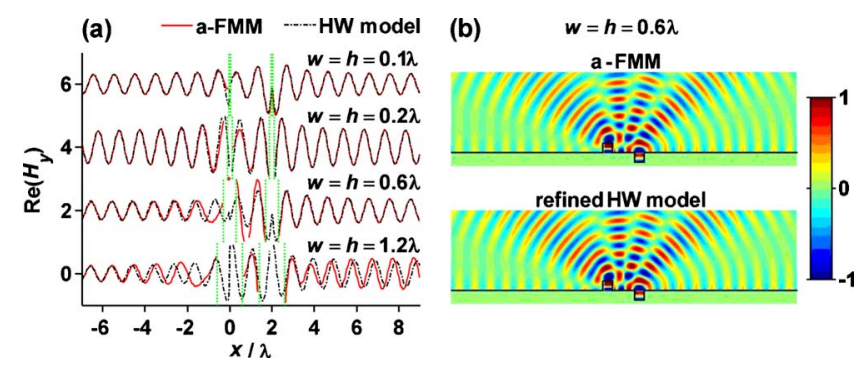

FIG. 6. (Color online) Enhanced accuracy of the HW model for large indentations. (a) Magnetic field scattered on the surface $(z$ $=0$ ) for ridge-groove doublets with several indentation dimensions, $w=h=0.1,0.2,0.6$, and $1.2 \lambda$. The predictions of the HW model [Eqs. (4) and (5)] and fully vectorial a-FMM computational results are shown by black dashed-dotted and red solid curves, respectively. The ridge and groove boundaries are shown with the vertical green dotted lines. (b) Magnetic field scattered by the ridge-groove doublet with large widths and heights, $w=h=0.6 \lambda$. The upper and lower plots are obtained with the a-FMM and the refined HW model, respectively. All the data are obtained for a doublet separation distance $d=2 \lambda$ and for $\lambda=1 \mu \mathrm{m}$. The incident field is a normally incident TM-polarized plane wave with a unitary magnetic field at the gold-air interface. The incident and specularly reflected plane waves have been removed for clarity.

tions: the first two correspond to the fields scattered by the two indentations under illumination by the plane wave [first term in Eq. (A5)], the third one is due to the illumination of the groove by a right-going SPP with an amplitude $P_{1}$ (the second term), and the last one is due to the illumination of the ridge by a left-going SPP with an amplitude $Q_{2}$ (the third term). In fact every contribution has been calculated when solving for the elementary scattering coefficients of every indentation.

The lower part in Fig. 6(b) shows the magnetic field scattered by the ridge-groove doublet, using the $P_{n}$ and $\mathrm{Q}_{n}$ coefficients calculated with the HW multiple-scattering model (as explained previously), but with the actual field scattered by every indentation. Although the calculation is performed for considerably large scatterers $(w=h=0.6 \lambda)$, the refined HW model is highly accurate for the near and far field (and even in the groove), as evidenced by a comparison with the scattered field calculated with the fully vectorial a-FMM (upper map).

\footnotetext{
*Author to whom correspondence should be addressed; liuht@nankai.edu.cn

${ }^{1}$ C. Genet and T. W. Ebbesen, Nature (London) 445, 39 (2007).

${ }^{2}$ M. E. Stewart, C. R. Anderton, L. B. Thompson, J. Maria, S. K. Gray, J. A. Rogers, and R. G. Nuzzo, Chem. Rev. 108, 494 (2008).

${ }^{3}$ S. P. Burgos, R. de Waele, A. Polman, and H. A. Atwater, Nature Mater. 9, 407 (2010).

${ }^{4}$ H. F. Schouten, N. Kuzmin, G. Dubois, T. D. Visser, G. Gbur, P. F. A. Alkemade, H. Blok, G. W. 't Hooft, D. Lenstra, and E. R. Eliel, Phys. Rev. Lett. 94, 053901 (2005).
}

${ }^{5}$ C. H. Gan, G. Gbur, and T. D. Visser, Phys. Rev. Lett. 98, 043908 (2007).

${ }^{6}$ L. Cai, G. Y. Li, Z. H. Wang, and A. S. Xu, Opt. Lett. 35, 127 (2010).

${ }^{7}$ L. Yin, V. K. Vlasko-Vlasov, J. Pearson, J. M. Hiller, J. Hua, U. Welp, D. E. Brown, and C. W. Kimball, Nano Lett. 5, 1399 (2005).

${ }^{8}$ F. López-Tejeira, S. G. Rodrigo, L. Martín-Moreno, F. J. GarcíaVidal, E. Devaux, T. W. Ebbesen, J. R. Krenn, I. P. Radko, S. I. Bozhevolnyi, M. U. González, J. C. Weeber, and A. Dereux, Nat. Phys. 3, 324 (2007). 
${ }^{9}$ I. I. Smolyaninov, D. L. Mazzoni, and C. C. Davis, Phys. Rev. Lett. 77, 3877 (1996).

${ }^{10}$ C. Genet, M. P. van Exter, and J. P. Woerdman, J. Opt. Soc. Am. A 22, 998 (2005).

${ }^{11}$ O. T. A. Janssen, H. P. Urbach, and G. W. 't Hooft, Opt. Express 14, 11823 (2006).

${ }^{12}$ A. Archambault, T. V. Teperik, F. Marquier, and J. J. Greffet, Phys. Rev. B 79, 195414 (2009).

${ }^{13}$ C. Genet, M. P. van Exter, and J. P. Woerdman, Opt. Commun. 225, 331 (2003).

${ }^{14}$ H. T. Liu and P. Lalanne, Nature (London) 452, 728 (2008).

${ }^{15}$ G. Gay, O. Alloschery, B. Viaris de Lesegno, C. O’Dwyer, J. Weiner, and H. J. Lezec, Nat. Phys. 2, 262 (2006).

${ }^{16}$ F. J. García de Abajo, Rev. Mod. Phys. 79, 1267 (2007).

${ }^{17}$ H. Raether, Surface Plasmons on Smooth and Rough Surfaces and on Gratings (Springer-Verlag, Berlin, 1988).

${ }^{18}$ R. Petit, Electromagnetic Theory of Gratings (Springer-Verlag, Berlin, 1980).

${ }^{19}$ A. Taflove, Computational Electrodynamics: The FiniteDifference Time-Domain Method (Artech House, Boston, MA, 1995).

${ }^{20}$ U. Fano, J. Opt. Soc. Am. 31, 213 (1941).

${ }^{21}$ U. Fano, Phys. Rev. 50, 573 (1936).

${ }^{22}$ P. Lalanne and J. P. Hugonin, Nat. Phys. 2, 551 (2006).

${ }^{23}$ W. Dai and C. M. Soukoulis, Phys. Rev. B 80, 155407 (2009).

${ }^{24}$ A. Y. Nikitin, S. G. Rodrigo, F. J. García-Vidal, and L. MartínMoreno, New J. Phys. 11, 123020 (2009).

${ }^{25}$ P. Lalanne, J. P. Hugonin, H. T. Liu, and B. Wang, Surf. Sci. Rep. 64, 453 (2009).

${ }^{26}$ The term "hybrid" is used to emphasize that the scattered field is composed of a bound part, the SPP of the flat metallic surface, and of a radiative part, the quasi-CW.

${ }^{27}$ E. D. Palik, Handbook of Optical Constants of Solids (Academic, New York, 1985), Part II.

${ }^{28}$ E. Silberstein, P. Lalanne, J. P. Hugonin, and Q. Cao, J. Opt. Soc. Am. A 18, 2865 (2001).

${ }^{29}$ J. P. Hugonin and P. Lalanne, J. Opt. Soc. Am. A 22, 1844
(2005).

${ }^{30}$ T. K. Gaylord and M. G. Moharam, Proc. IEEE 73, 894 (1985).

${ }^{31}$ More precisely, the mixing ratio mainly depends on the metal permittivity $\varepsilon_{m}$ that is itself varying with the frequency, $H_{\mathrm{CW}}^{+}(\lambda, 0) / H_{\mathrm{SP}}^{+}(\lambda, 0) \approx-i^{1 / 2}(2 \pi)^{-1}\left(\varepsilon_{d}\right)^{-3 / 4}\left(\varepsilon_{m}\right)^{1 / 2}$ for instance, and details are reported in Ref. 25.

${ }^{32}$ X. Y. Yang, H. T. Liu, and P. Lalanne, Phys. Rev. Lett. 102, 153903 (2009).

${ }^{33}$ Normal modes are defined as waveguide modes that obey an exponential propagation rule $\exp \left(i k_{0} n_{\mathrm{eff}} z\right)$ along the invariant $z$ direction of the waveguide, see details in Refs. 34 and 35.

${ }^{34}$ C. Vassallo, Optical Waveguide Concepts (Elsevier, Amsterdam, 1991).

${ }^{35}$ H. A. Haus, Waves and Fields in Optoelectronics (Prentice-Hall International, London, 1984).

${ }^{36}$ Note that a similar argument has been recently applied in Ref. 32 to analyze the CW-to-SPP conversion.

${ }^{37}$ H. T. Liu, P. Lalanne, X. Y. Yang, and J. P. Hugonin, IEEE J. Sel. Top. Quantum Electron. 14, 1522 (2008).

${ }^{38}$ P. Lalanne, J. P. Hugonin, and J. C. Rodier, J. Opt. Soc. Am. A 23, 1608 (2006).

${ }^{39}$ E. L. Tan, J. Opt. Soc. Am. A 19, 1157 (2002).

${ }^{40}$ M. Mansuripur, J. Opt. Soc. Am. A 6, 786 (1989).

${ }^{41}$ T. W. Ebbesen, H. J. Lezec, H. F. Ghaemi, T. Thio, and P. A. Wolff, Nature (London) 391, 667 (1998).

${ }^{42}$ L. Martín-Moreno, F. J. García-Vidal, H. J. Lezec, K. M. Pellerin, T. Thio, J. B. Pendry, and T. W. Ebbesen, Phys. Rev. Lett. 86, 1114 (2001).

${ }^{43}$ H. T. Liu and P. Lalanne, J. Opt. Soc. Am. A (to be published). The authors present a comprehensive microscopic model of extraordinary optical transmission, which analyzes the EOT phenomenon from the point of view of the hybrid-wave model, emphasizing the impact of the metal permittivity (including the perfect-conductor case).

${ }^{44}$ T. Søndergaard and S. I. Bozhevolnyi, Phys. Rev. B 67, 165405 (2003).

${ }^{45}$ R. E. Collin, IEEE Antennas Propag. Mag. 46, 64 (2004). 